\title{
INTRODUCTION DE LA TORTUE DE FLORIDE TRACHEMYS SCRIPTA EN FRANCE. UN NOUVEAU COMPÉTITEUR POUR LES ESPĖCES DE TORTUES D'EAU DOUCE EUROPÉENNES.
}

\author{
J. SERVAN (1) et C. ARVY (2)
}

(1) Muséum National d'Histoire Naturelle - Institut d'Ecologie et de Gestion de la Biodiversité, Laboratoire d'Evolution des Systèmes Naturels et Modifiés, 36 rue Geoffroy St-Hilaire, 75005 Paris, France.

(2) Muséum National d'Histoire Naturelle, Laboratoire de Biologie des Invertébrés Marins et Malacologie, 57 rue Cuvier, 75005 Paris, France.

\section{RÉSUMÉ}

Des importations importantes de Trachemys scripta en France comme animal de compagnie ont lieu depuis plus de 10 ans. De plus en plus souvent, des animaux sont abandonnés dans la nature, de sorte que l'espèce est présente dans presque tous les départements français et se reproduit dans trois régions où vit Emys orbicularis. Une analyse comparée de plusieurs aspects de leur biologie montre un large recouvrement de leur niche écologique.

THE INTRODUCTION OF TRACHEMYS SCRIPTA IN FRANCE. A NEW COMPETITOR FOR THE EUROPEAN POND TURTLES.

\section{ABSTRACT}

Trachemys scripta has been massively imported into France as a pet animal for ten years. More and more animals are released in nature so that now Trachemys scripta is widely distributed in France and reproduction has been recorded in three places where Emys orbicularis is living. A comparison of some aspects of their life history shows a wide overlap in their ecological niches.

\section{INTRODUCTION}

La présence d'espèces en dehors de leur aire naturelle de répartition à l'époque historique s'explique essentiellement par leur transport par des hommes. Le développement du commerce de denrées alimentaires entre les continents a pris une importance majeure depuis les grandes découvertes du XV-XVllè siècle. Puis les transferts d'espèces se firent à des fins de production purement commerciale. Parmi les espèces volontairement transférées, certaines se sont acclimatées et naturalisées, en échappant au contrôle des hommes, et ont perturbé les faunes et les flores locales. 
Aux USA, le commerce de la tortue de Floride Trachemys scripta s'est rapidement développé après la seconde guerre mondiale. Vers 1960, quelques 150 entreprises produisaient environ 13 millions de petites tortues par an, principalement pour le marché américain (10 millions) et canadien ( 3 millions) (SALZBERG, comm. pers.). A cette même époque, sont apparus les premiers rapports sur des enfants souffrant de salmonellose et il fallut plus d'une dizaine d'années pour que les scientifiques mettent en relation ces nombreux cas avec la possession d'une tortue aquatique, puis pour préparer un texte de loi promulgué en 1975, à l'initiative de l'U.S. Food and Drug Administration, interdisant la vente de tortues d'eau douce d'une taille inférieure à quatre inches (environ $12 \mathrm{~cm}$ ). En conséquence, de nombreuses entreprises fermèrent, et les autres se tournèrent vers l'exportation. En 1993, une cinquantaine d'entreprises exportaient plus de six millions de tortues de Floride par an. En France, pendant les 10 dernières années, les importations (Tableau I) ont varié de 0 en 1992 (interdiction d'importation) à 1878000 en 1989-1990, soit en moyenne plus de 400000 tortues par an.

\section{Tableau I}

Importations de Trachemys scripta en France (d'après WARWICK, 1991; Reptile Protection Trust, comm. pers.).

Table I

Importations of Trachemys scripta in France (from WARWICK, 1991 ; Reptile Protection Trust, unedited data).

\begin{tabular}{|l|c|c|c|c|c|c|c|c|c|}
\hline Année & 1985 & 1986 & 1987 & 1988 & $1989 / 90$ & 1991 & 1992 & 1993 & 1994 \\
\hline Nombre & 170000 & 200000 & 190000 & 420000 & 1878809 & 680000 & 0 & 530000 & 170000 \\
\hline
\end{tabular}

Les observations de tortues de Floride dans le milieu naturel, liées à l'abandon des animaux par leurs propriétaires, ont d'abord été anecdotiques puis elles ont été de plus en plus nombreuses dans les années 1980.

Les résultats de deux enquêtes sur la répartition de la tortue de Floride en France sont présentés ci-dessous, ainsi qu'une comparaison de diverses données écologiques concernant la tortue de Floride Trachemys scripta elegans et la cistude d'Europe Emys orbicularis orbicularis.

\section{RÉPARTITION DE LA TORTUE DE FLORIDE EN FRANCE}

Deux enquêtes, menées en 1994-1995 par le Ministère de l'Environnement/CSP et en 1995 par le collectif tortue de Floride, montrent que des tortues de Floride sont présentes dans le milieu naturel dans tous les départements métropolitains à l'exception de l'Ariège, des Hautes Pyrénées et du Tarn et Garonne, pour lesquels nous ne disposons d'aucun renseignement. L'espèce est donc présente dans tous les grands bassins hydrographiques. $59 \%$ des 722 observations de la seconde enquête ont été effectuées dans des lacs et étangs, $16 \%$ dans des cours d'eau, le reste se répartissant dans d'autres types de milieux. Un seul individu a été observé dans $48 \%$ des cas et deux à dix individus dans $43 \%$ des observations. $77 \%$ des observations concernaient des individus dont la longueur de la carapace dépassait $10 \mathrm{~cm}$, alors que seulement $23 \%$ des témoignages rapportent des observations d'animaux ne dépassant pas $10 \mathrm{~cm}$. Trois cas de reproduction complète sont signalés : l'un près de Toulouse, un second près d'Alès et le troisième près de Tours.

\section{COMPARAISON ENTRE LES DEUX ESPÈCES}

Parmi les 15 sous-espèces de Trachemys scripta (ERNST, 1990), la sous-espèce elegans est de loin la plus importée sur le marché français. Trois des 11 sous-espèces d'Emys orbicularis (FRITZ, 1992, 1995) sont présentes en France : E.o. orbicularis dans le Centre, 
E.o. galloitalica de Nice à Biarritz et E.o. lanzai en Corse. La biologie d'E.o. orbicularis est la mieux connue, c'est pourquoi nous la comparons à celle de $T$. s. elegans.

Les deux espèces sont des tortues aquatiques fréquentant diverses catégories de zones humides telles que mares, marais, cours d'eau, étangs et lacs, mais $T$. s. elegans présente une activité terrestre plus importante que la cistude (CAGLE, 1946; ERNST et BARBOUR, 1989; MINYARD, 1947 ; ROLLINAT, 1934 ; SERVAN, 1986, 1988). La longueur de la carapace de la tortue de Floride est en moyenne plus grande que celle de la cistude, aussi bien pour les mâles (156 mm contre $136 \mathrm{~mm}$ ) que pour les femelles (204 mm contre $147 \mathrm{~mm}$ ) (CAGLE, 1950 ; SERVAN, 1988). Cependant, la longueur minimale des mâles de tortue de Floride à la maturité sexuelle est inférieure, ce qui s'explique par la précocité de la maturité de la tortue de Floride: deux à cinq ans pour T. s. elegans (CAGLE, 1950), contre six à seize ans pour E.o. orbicularis.

La densité de la tortue de Floride aux USA atteint couramment, dans les secteurs favorables, plus de 100 individus par hectare (CAGLE, 1950), alors que la cistude ne dépasse guère 15 individus par hectare. Des densités très élevées sont relevées dans des cas extrêmes et toujours pour des surfaces très réduites : plus de 1000 cistudes par hectare dans deux mares de 65 et $90 \mathrm{~m}^{2}$ (NAULLEAU, 1991) et plus de 3000 tortues de Floride par hectare dans une petite mare (CAGLE, 1950).

La proportion de juvéniles est faible pour les deux espèces : $31 \%$ pour la cistude (SERVAN, 1988) et 19 à $31 \%$ pour la tortue de Floride (CAGLE, 1942, 1950). Le sex-ratio chez les adultes est dévié pour la cistude en faveur des femelles (47\% de mâles) en Brenne tandis que des valeurs allant de 0,69 à 1,49 sont citées pour la tortue de Floride (CAGLE, 1942, 1950 ; PARKER, 1984).

Les deux espèces sont principalement carnivores, cependant la tortue de Floride consomme une proportion croissante de végétaux au fur et à mesure de sa croissance et jusqu'à $60 \%$ pour les animaux les plus grands (HART, 1983).

La ponte a lieu entre avril et juin aux USA pour $T$. $s$. elegans et entre mai et juillet pour E.o. orbicularis. Le nombre moyen d'oeufs par ponte pour la cistude est proche de 8,5 (SERVAN et PIEAU, 1984 ; ROLLINAT, 1934) et pour la tortue de Floride il varie de 7 à 10,5 (CAGLE, 1950). Le poids à la naissance est de $4,8 \mathrm{~g}$ pour les cistudes (SERVAN et PIEAU, 1984) et de $8,1 \mathrm{~g}$ pour les tortues de Floride (CAGLE, 1950).

\section{DISCUSSION}

La présence de Trachemys scripta elegans dans la quasi-totalité des départements métropolitains, avec parfois des observations sur plusieurs années, montre que cette espèce est acclimatée. Les quelques cas de reproduction réussie montrent que la naturalisation est possible, même si le processus semble à peine engagé aujourd'hui. La tortue de Floride arrive dans un espace que deux espèces de tortues, Emys orbicularis et la rare Mauremys caspica, ont colonisé depuis la fin des glaciations. Une compétition entre les espèces semble inéluctable si aucune mesure n'est prise pour limiter la présence de $T$. scripta. La comparaison des paramètres biologiques (Tableau II) montre que la tortue de Floride est plus grande, a une reproduction plus précoce, des oeufs plus gros, des jeunes plus lourds et des populations plus nombreuses que la cistude. D'un point de vue alimentaire, la cistude est strictement carnivore tandis que le régime alimentaire de la tortue de Floride comporte une proportion de végétaux importante pour les animaux les plus grands. Le potentiel reproducteur est de même grandeur pour les deux espèces. Théoriquement, $T$. s. elegans possède des avantages certains pour une compétition avec la cistude. Cependant, de nombreuses inconnues subsistent : comment vont s'exprimer les potentialités de la tortue de Floride dans les conditions qu'offre l'Europe en matière de climat, de ressources trophiques... Ces conditions vont-elles favoriser ou défavoriser l'espèce nouvelle? D'autre part, comment va réagir la cistude à cette compétition? La régression, à la fois naturelle et liée aux activités humaines, de cette espèce va-t-elle être accélérée ? Seules des études ultérieures permettront de répondre à ces questions. 


\section{Tableau II}

Caractéristiques écologiques de Trachemys scripta et d'Emys orbicularis.

Table II

Ecological characteristics of Trachemys scripta and Emys orbicularis.

\begin{tabular}{|l|l|l|}
\hline & T. scripta & E. orbicularis \\
\hline Taille & Plus grande & \\
\hline Alimentation & Omnivore & Carnivore \\
\hline Densité & Plus élevée & \\
\hline Oeufs & Plus gros & \\
\hline Jeune & Plus grand & \\
\hline Reproduction & Plus précoce & \\
\hline
\end{tabular}

Si la tortue de Floride est dominante en quantités vendues, elle n'est pas la seule espèce présente sur le marché : sept autres espèces américaines ont été exportées dans le monde à plus de 10000 exemplaires entre 1989 et 1994. La France n'est pas le seul pays d'exportation, comme en témoigne le nombre d'états où la tortue de Floride s'est acclimatée : Afrique du Sud, Arabie, Bahrein, Brésil, Espagne, Grande Bretagne, Israël, Italie, Japon, Malaisie, Panama, Singapour, Sri Lanka et Thailande (BOUR, comm. pers.; MOLL, 1995 ; SALZBERG, comm. pers.). En France, en dehors de la métropole, elle est déjà naturalisée en Guadeloupe, Guyane, Martinique, Polynésie et Réunion (BOUR, comm. pers. ; DUPRE, comm. pers.).

\section{REMERCIEMENTS}

Cette étude a été soutenue par le Collectif Tortue de Floride : ASPAS, FNE, SHF, SOPTOM et SPA.

\section{BIBLIOGRAPHIE}

CAGLE F.R., 1942. Turtle population of southern Illinois. Copeia, 1942, 155-162.

CAGLE F.R., 1946. The growth of the slider turtle, Pseudemys scripta elegans. Amer. Midl. Nat., 36, 685-739.

CAGLE F.R., 1950. The life history of the slider turtle, Pseudemys scripta troostii (Hoolbrook). Ecol. monographs, 20, 31-54.

ERNST C.H., 1990. Systematic, taxonomy, variation and geographic distribution of the slider turtle in GIBBONS J.W., The slider turtle. Life history and ecology of the slider turtle, 5767, Smithonian Institution Press, Washington, D.C.

ERNST C.H., BARBOUR R.W., 1989. Turtles of the world. Smithonian Institution Press, Washington D.C., $313 \mathrm{p}$.

FRITZ U., 1992. Zur innerartlichen Variabilität von Emys orbicularis (Linnaeus, 1758). 2. Variabilität in Osteuropa und Redefinition von Emys orbicularis orbicularis (Linnaeus, 1758) und E. o. hellenica (Valenciennes, 1832). Zool. Abh. Mus. Tierkd. Dresden, 48 (13), $37-77$. 
FRITZ U., 1995. Zur innerartlichen Variabilität von Emys orbicularis (Linnaeus, 1758). Taxonomie in Mittel-Westeuropa, auf Korsika, Sardinien, der Apenninen-Halbinsel und Sicilien und Unterartengruppen von Emys orbicularis (Reptilia: Testudines: Emydidae). Zool. Abh. Mus. Tierkd. Dresden, 48 (13), 185-242.

HART D.R., 1983. Dietary and habitat shift with size of red-eared turtles (Pseudemys scripta) in a southern Louisiana population. Herpetologica, 39, 285-290.

MINYARD V., 1947. The food habits of the slider turtle Pseudemys scripta troostii. Master's thesis Tulane.

MOLL E.O., 1995. The turtle Trachemys scripta and the pet trade. ALIENS, (2), 3.

NAULLEAU G., 1991. Adaptations écologiques d'une population de cistudes (Emys orbicularis L.) (Reptilia, Chelonii) aux grandes variations de niveau d'eau et à l'assèchement naturel du milieu aquatique fréquenté. Bull. Soc. Herp. Fr., 58, 11-19.

PARKER W.S., 1984. Immigration and dispersal of the slider turtles Pseudemys scripta in Mississippi farm pond. Am. Midl. Nat., 112, 280-293.

ROLLINAT R., 1934. La vie des reptiles dans la France centrale. Delagrave ed., 343 p.

SERVAN J., 1986. Répartition de la cistude d'Europe, Emys orbicularis, dans les marais de l'Ouest de la France. 111 ème Congrès des Sociétés Savantes, Poitiers, sciences (II), 195-202.

SERVAN J., 1988. La cistude d'Europe, Emys orbicularis, dans les étangs de Brenne, France. Mésogée, 48, 91-95.

SERVAN J., PIEAU C., 1984. La cistude d'Europe (Emys orbicularis) : mensurations d'oeufs et de jeunes individus. Bull. Soc. Herp. Fr., 31, 20-26. 